\title{
ESTABELECIMENTO DE PASTAGEM CONSORCIADA COM APLICAÇÃO DE CALCÁRIO, FÓSFORO E GESSO ${ }^{1}$
}

\author{
Establishment of mixed pasture with application of limestone, gypsum and phosphorus
}

\author{
Eduardo Eustáquio Mesquita ${ }^{2}$, Dilermando Miranda da Fonseca ${ }^{3}$, \\ José Cardoso Pinto ${ }^{4}$, Domício do Nascimento Junior ${ }^{5}$, Odilon Gomes Pereira ${ }^{3}$, \\ Victor Hugo Alvarez Venegas ${ }^{6}$, Luciano de Melo Moreira ${ }^{7}$
}

\section{RESUMO}

Em solo de baixa fertilidade, sob pastagem natural degradada, pertencente à Universidade Federal de Viçosa - Viçosa (MG), foi estabelecida uma pastagem de Brachiaria decumbens consorciada com Stylosanthes guianensis cv. Mineirão, para avaliar os efeitos de doses de calcário $(1.175,2.350,3.525$ e $4.700 \mathrm{~kg} / \mathrm{ha}$, referentes a 25, 50, 75 e $100 \%$ da quantidade de calcário recomendada-QC), gesso agrícola $(0,230,940,1.880$ e $2.820 \mathrm{~kg} / \mathrm{ha}$, referentes à substituição de $0,3,0,12,5,25,0$ e 37,5\% do CaO do calcário pelo $\mathrm{CaO}$ do gesso) e de $\mathrm{P}_{2} \mathrm{O}_{5}\left(50,100,150,200\right.$ e $250 \mathrm{~kg} / \mathrm{ha}$ ) sobre o pH do solo; os teores de $\mathrm{P}, \mathrm{K}$ e $\mathrm{S}$ disponíveis e de $\mathrm{Ca}^{2+}, \mathrm{Mg}^{2+}$, $\mathrm{Al}^{3+}, \mathrm{H}+\mathrm{Al}$; a soma de bases (SB); a CTC efetiva (t) e a pH 7,0 (T); o índice de saturação por bases (V) e por $\mathrm{Al}$ (m); a produção de matéria seca, densidade de perfilhos e a altura de plantas da braquiária; e a porcentagem da leguminosa na pastagem. A substituição do $\mathrm{CaO}$ do calcário pelo $\mathrm{CaO}$ do gesso elevou a porcentagem do estilosantes na pastagem e os teores de $\mathrm{S}_{-} \mathrm{SO}_{4}{ }^{2-}$ e de $\mathrm{Al}^{3+}$, nas camadas de 0-15 e de 15-30 cm, e reduziu os teores de $\mathrm{Ca}^{2+}$ e de $\mathrm{Mg}^{2+}$, na camada de 0-15 cm. As doses crescentes de $\mathrm{P}_{2} \mathrm{O}_{5}$ aumentaram os teores de $\mathrm{P}$ (Mehlich-1) na camada de $0-15 \mathrm{~cm}$ do solo e favoreceram o perfilhamento e a altura da gramínea, reduzindo a porcentagem da leguminosa na pastagem.

Termos para indexação: Leguminosa, gramínea, cálcio trocável, fósforo e enxofre disponíveis, composição botânica, densidade de perfilhos.

\section{ABSTRACT}

At low fetility soil, in degraded natural pasture, a field trial was carried out at the Federal University of Viçosa, located at Viçosa - MG, was established the pasture of Brachiaria decumbens in mixture with Stylosanthes guianensis cv. Mineirão to evaluate the effect of limestone doses $(1.175,2.350,3.525$ and $4.700 \mathrm{~kg} / \mathrm{ha}$, equivalent 25, 50, 75 and $100 \%$ of limestone necessity), gypsum doses $(0,230,940,1.880$ e $2.820 \mathrm{~kg} / \mathrm{ha}$ equivalent the substitution of $0,3,0,12,5,25,0$ and $37,5 \%$ of limestone $\mathrm{CaO}$ by gypsum $\mathrm{CaO})$ and $\mathrm{P}_{2} \mathrm{O}_{5}$ doses $(50,100,150,200$ and $250 \mathrm{~kg} / \mathrm{ha}$ ) on the $\mathrm{pH}$; availables $\mathrm{P}, \mathrm{S}$ and $\mathrm{K}$; exchangeables $\mathrm{Ca}^{2+}, \mathrm{Mg}^{2+}, \mathrm{Al}^{3+}, \mathrm{H}+\mathrm{Al}$; sum of bases (SB); effetive CEC ( $\mathrm{t}$ ) and CEC determined in $\mathrm{pH} 7,0(\mathrm{~T})$; base saturation (V); aluminum saturation (m) and dry matter production, tillers number and height of brachiaria grass and legume percent. The substitution of the limestone $\mathrm{CaO}$ by gypsum $\mathrm{CaO}$ increased the percent of stylosanthes, available $\mathrm{S}$ and $\mathrm{Al}^{3+}$, in $0-15$ and $15-30 \mathrm{~cm}$ soil layer, and decreased $\mathrm{Ca}^{2+}$ and $\mathrm{Mg}^{2+}$, in 0-15 $\mathrm{cm}$ soil layer. The $\mathrm{P}$ doses increased the available $\mathrm{P}$ (Mehlich 1) in 0-15 $\mathrm{cm}$ soil layer, but decreased the legume percent in the pasture.

INDEX TERMS: Legume, grass, exchangeable calcium, availables phosphorus and sulphur, botanical composition, tiller density.

(Recebido para publicação em 24 de julho de 2002 e aprovado em 11 de novembro de 2002)

\footnotetext{
1. Parte da Tese de doutorado em Zootecnia do primeiro autor, apresentada à UFV - Departamento de Zootecnia - 36571-000 Viçosa, MG

2. Recém-Doutor em Zootecnia, Universidade Federal de Lavras/UFLA - Caixa Postal 37 - 37200-000 - Lavras, MG, bolsista do CNPq. e_mesquita@bol.com.br

3. Professor Adjunto do Departamento de Zootecnia, UFV.

4. Professor Adjunto do Departamento de Zootecnia, UFLA

5. Professor Titular do Departamento de Zootenia, UFV.

6. Professor Titular do Departamento de Solos, UFV.

7. Estudante do Programa de pós-graduação em Zootecnia, UFV.
} 


\section{INTRODUÇÃO}

As pastagens naturais da região da Zona da Mata de Minas Gerais são caracterizadas por solos com topografia acidentada, elevada acidez, baixos teores de fósforo (P), específicos arranjos de espécies (PACHECO et al., 1987) e predominância do capimgordura (Melinis minutiflora Beauv.) (SANTOS et al., 1998), espécie de maior participação na dieta dos animais (DIOGO et al., 1995). Introduções de novas espécies forrageiras mais produtivas, por meio de métodos adequados de preparo de solo, protegendo-o da erosão, exigem suprimento adequado de nutrientes.

A introdução de Brachiaria decumbens Stapf e Stylosanthes guianensis (Aubl.) Sw. var. vulgaris cv. Mineirão em consórcio pode suprir parte do $\mathrm{N}$ requerido para a manutenção da produção; todavia, o P é o principal nutriente para o estabelecimento das espécies forrageiras e sua disponibilidade normalmente é aumentada pela prática da calagem. Em áreas declivosas, a incorporação de calcário com preparo convencional do solo é dificultada; assim, a aplicação de gesso agrícola no sulco de semeadura pode ser uma alternativa para o enriquecimento em bases nas camadas mais profundas do solo e proporcionar maior aprofundamento das raízes. Michalk et al. (1998) demonstraram que o sulcamento, sem aração e gradeação do solo, e a aplicação de $28 \mathrm{~kg} / \mathrm{ha}$ de $\mathrm{P}$ no sulco foram suficientes para o estabelecimento de várias cultivares de Stylosanthes em solo de baixa fertilidade.

Além de elevar os teores de $\mathrm{Ca}^{2+}, \mathrm{S}_{-} \mathrm{SO}_{4}{ }^{2}$, $\mathrm{Mg}^{2+}$ e $\mathrm{K}$ disponível nas camadas mais profundas, a adição de gesso reduz o efeito do alumínio e eleva o pH do solo (WANG et al., 1999); embora, muitas vezes, a alteração no $\mathrm{pH}$ é pouco expressiva e dependente do tipo de solo. Segundo Rechcigl e Mislevy (1997), quantidades maiores de gesso podem aumentar a concentração de sais, especialmente sais de cálcio, reduzindo o $\mathrm{pH}$ do solo.

Em consórcios de gramíneas e leguminosas, as adubações devem direcionar-se para essas últimas, pois essa é uma das maneiras de favorecer a leguminosa, geralmente mais exigente que a gramínea (ZIMMER et al., 1994). Leguminosas como Pueraria phaseoloides, Centrosema pubescens, Arachis pintoi (SANTOS et al., 1999) e Stylosanthes guinenesis cv.
Cook (GONÇALVES et al., 1997), reconhecidamente exigentes em $\mathrm{P}$, foram beneficiadas com a aplicação de P. Entretanto, espécies de baixa exigência em $\mathrm{P}$ e tolerante à baixa acidez do solo, como o estilosantes cv. Mineirão (EMBRAPA, 1993), respondem menos à aplicação do elemento do que a gramínea. Nessa condição, Andrade e Karia (2000) ressaltaram que doses acima de $100 \mathrm{~kg} / \mathrm{ha}$ de $\mathrm{P}_{2} \mathrm{O}_{5}$, mais calagem, poderiam beneficiar o crescimento da gramínea, aumentar a sua competição por luz e nutrientes e reduzir a persistência da leguminosa na pastagem. Em consórcio de $S$. guianensis cv. Cook e B. decumbens, apenas $50 \mathrm{~kg} / \mathrm{ha}$ de $\mathrm{P}_{2} \mathrm{O}_{5}$, aplicados em Latossolo de baixa fertilidade, foram suficientes para aumentar em $32 \%$ a produção de MS das espécies; porém, não se constatou efeito do $\mathrm{P}$ sobre o percentual da leguminosa na composição botânica (GONÇALVES et al., 1997).

Este trabalho foi desenvolvido para avaliar, em solo de baixa fertilidade da região de Viçosa (MG), o efeito doses de calcário, calcário mais gesso e fósforo sobre a melhoria do ambiente radicular e a porcentagem de Stylosantes guianensis cv. Mineirão na pastagem consorciada com Brachiaria decumbens cv. Basilisk.

\section{MATERIAL E MÉTODOS}

O trabalho foi conduzido em área declivosa de pastagem natural degradada pertencente ao Departamento de Zootecnia da Universidade Federal de Viçosa. Nessa área, introduziram-se em consórcio as espécies Brachiaria decumbens Stapf cv. Basilisk e Stylosanthes guianensis (Aubl.) Sw. var. vulgaris cv. Mineirão. A cidade de Viçosa (MG) localiza-se na Zona da Mata mineira, altitude de $651 \mathrm{~m}$, nas coordenadas de $20^{\circ} 45^{\prime}$ de latitude sul e $42^{\circ} 51^{\prime}$ de longitude oeste. O clima, pelo sistema de Köppen, é do tipo Cwa, com precipitação anual média em torno de $1.340 \mathrm{~mm}$, umidade relativa do ar média de $80 \%$ e as temperaturas médias máxima e mínima de 22,1 e $15^{\circ} \mathrm{C}$, respectivamente. As características químicas e físicas do solo (Tabela 1), classificado como Latossolo VermelhoAmarelo distrófico, amostrado na área experimental em duas profundidades, antes da aplicação dos tratamentos, foram determinadas nos laboratórios do Departamento de Solos da UFV. 
TABELA 1 - Características químicas e físicas de amostras de solo coletadas em duas profundidade, na região de Viçosa, MG.

\begin{tabular}{|c|c|c|}
\hline \multirow{2}{*}{ Características } & \multicolumn{2}{|c|}{ Profundidades (cm) } \\
\hline & $0-15$ & 15-30 \\
\hline pH em água $(1: 2,5)$ & 5,0 & 4,9 \\
\hline $\mathrm{P}\left(\right.$ Mehlich-1) - mg/dm ${ }^{3}$ & 3,8 & 1,0 \\
\hline $\mathrm{K}\left(\right.$ Mehlich-1) $-\mathrm{mg} / \mathrm{dm}^{3}$ & 22,0 & 22,0 \\
\hline $\mathrm{Ca}^{2+}(\mathrm{KCl} 1 \mathrm{~mol} / \mathrm{L})-\mathrm{cmol}_{\mathrm{c}} / \mathrm{dm}^{3}$ & 0,6 & 0,3 \\
\hline $\mathrm{Mg}^{2+}(\mathrm{KCl} 1 \mathrm{~mol} / \mathrm{L})-\mathrm{cmol}_{\mathrm{c}} / \mathrm{dm}^{3}$ & 0,1 & 0,1 \\
\hline $\mathrm{Al}^{3+}(\mathrm{KCl} 1 \mathrm{~mol} / \mathrm{L})-\mathrm{cmol}_{\mathrm{c}} / \mathrm{dm}^{3}$ & 0,5 & 0,4 \\
\hline $\mathrm{S}-\mathrm{SO}_{4}{ }^{2-}\left(\mathrm{Ca}\left(\mathrm{H}_{2} \mathrm{PO}_{4}\right)_{2} \mathrm{em} \mathrm{HOAc}\right)-\mathrm{mg} / \mathrm{dm}^{3}$ & 18,0 & 19,0 \\
\hline $\mathrm{H}+\mathrm{Al}($ acetato de cálcio $-0,5 \mathrm{~mol} / \mathrm{L})-\mathrm{cmol}_{\mathrm{c}} / \mathrm{dm}^{3}$ & 4,0 & 3,6 \\
\hline Soma de Bases $\left(\mathrm{cmol}_{\mathrm{c}} / \mathrm{dm}^{3}\right)$ & 0,8 & 0,5 \\
\hline CTC efetiva $\left(\mathrm{cmol}_{\mathrm{c}} / \mathrm{dm}^{3}\right)$ & 1,3 & 0,9 \\
\hline $\mathrm{T}\left(\mathrm{CTC}\right.$ total $\left.\mathrm{pH}=7,0 ; \mathrm{em} \mathrm{cmol}_{\mathrm{c}} / \mathrm{dm}^{3}\right)$ & 4,8 & 4,1 \\
\hline V (Saturação por bases; em \%) & 17,0 & 12,0 \\
\hline m (Saturação por alumínio, em \%) & 62,5 & 80,0 \\
\hline Areia $(\%)$ & 29,0 & 26,0 \\
\hline Silte $(\%)$ & 29,0 & 24,0 \\
\hline Argila (\%) & 42,0 & 50,0 \\
\hline
\end{tabular}

O delineamento experimental foi em blocos ao acaso, com três repetições. Os tratamentos consistiram de três fatores, sendo quatro doses de calcário dolomítico $(1.175,2.350,3.525$ e $4.700 \mathrm{~kg} / \mathrm{ha}$, referentes a 25 , 50,75 e $100 \%$ da quantidade recomendada de calcário QC), cinco doses gesso (0, 230, 940, 1880 e 2820 $\mathrm{kg} / \mathrm{ha}$, referentes à substituição de 0, 3,0, 12,5, 25,0 e $37,5 \%$ do $\mathrm{CaO}$ do calcário pelo $\mathrm{CaO}$ do gesso, na dose de $100 \%$ da QC) e cinco doses de fósforo $(50,100,150$, 200 e $250 \mathrm{~kg} / \mathrm{ha}$ de $\mathrm{P}_{2} \mathrm{O}_{5}$ ), sob a forma de superfosfato triplo. Os fatores e seus respectivos níveis foram arranjados na forma de matriz. Cada fator foi analisado separadamente, fixando valores de referência para os outros dois fatores. Os valores de referência foram calculados de acordo com a CFSEMG (1999), ou seja, $100 \mathrm{~kg} / \mathrm{ha}$ de $\mathrm{P}_{2} \mathrm{O}_{5}, 230 \mathrm{~kg} / \mathrm{ha}$ de gesso e 4.700 $\mathrm{kg} / \mathrm{ha}$ de calcário dolomítico. A QC foi obtida pelo método de saturação por bases para elevar $\mathrm{V}$ a $60 \%$ na camada de 0-30 cm, pela seguinte fórmula: $\mathrm{QC}=$
[ $(0,6 \mathrm{~T}-\mathrm{SB}) 0,3 /(0,2 \times 0,65)]$; em que QC é a quantidade, em t/ha, de um calcário com PRNT de $65 \%$, para correção da camada de 0-30 $\mathrm{cm}$; e $\mathrm{T}=4,4$ $\mathrm{cmol}_{\mathrm{c}} / \mathrm{dm}^{3}$ e $\mathrm{SB}=0,6 \mathrm{cmol}_{\mathrm{c}} / \mathrm{dm}^{3}$ são, respectivamente, as médias da capacidade de troca de cátions e da soma de bases do solo, nas camadas de 0-15 e 15-30 cm (Tabela 1).

Efetuou-se a abertura de nove sulcos/parcela (25 $\mathrm{cm}$ de largura $\mathrm{x} 20 \mathrm{~cm}$ de profundidade). Nas parcelas (9,0 m de largura x $10 \mathrm{~m}$ de comprimento), usou-se sulcador reversível tracionado por animais. Em todas as parcelas, foram feitas adubações básicas com 40, 20, 20 e $0,53 \mathrm{~kg} /$ ha de $\mathrm{K}_{2} \mathrm{O}, \mathrm{ZnSO}_{4}$, bórax e $\mathrm{Na}_{2} \mathrm{MoO}_{4}$, respectivamente, aplicadas no sulco de semeadura. Na aplicação do calcário e do gesso, considerou-se a área correspondente ao sulco de semeadura $\left(2,5 \mathrm{~m}^{2} /\right.$ sulco.parcela ou $2.500 \mathrm{~m}^{2} / \mathrm{ha}$ ).

Utilizaram-se para a semeadura 4 e $2 \mathrm{~kg} / \mathrm{ha}$ de sementes puras viáveis de braquiária e estilosantes, res- 
pectivamente. Distribuíram-se as sementes nos sulcos em segmentos de 1,42 m, alternando segmentos de gramínea e leguminosa no mesmo sulco, de modo que as sementes da gramínea e da leguminosa ficassem separadas, começando e terminando a semeadura com a leguminosa. Com a semeadura separada das espécies, objetivou-se favorecer o estabelecimento inicial da leguminosa, que reconhecidamente é menos competitiva que a gramínea. Determinou-se a porcentagem da leguminosa na pastagem com base na sua produção de MS em relação à produção de MS total das espécies. A avaliação da produção de MS foi feita separadamente para cada espécie por meio de três cortes a cada ano, com intervalos de 48 dias, realizados a $10 \mathrm{~cm}$ do nível do solo. As amostras de forragens foram secas em estufa a $65^{\circ} \mathrm{C}$ até atingir peso constante.

Vinte e sete meses após, foram retiradas, ao acaso, vinte amostras simples de solo em cada parcela, sendo 10 nas linhas de semeadura e 10 nas entrelinhas, que deram origem a uma amostra composta nas camadas de 0-15 e 15-30 cm de profundidade. Prevendo-se a mobilização de nutrientes das linhas para as entrelinhas, em razão da acentuada declividade do solo, retiraram-se amostras de solo também nas entrelinhas.

Nas amostras, foram determinados: $\mathrm{pH}$ em $\mathrm{H}_{2} \mathrm{O}$ relação $1: 2,5 ; \mathrm{Ca}^{2+}, \mathrm{Mg}^{2+}$ e $\mathrm{Al}^{3+}$ extraídos pelo $\mathrm{KCl}(1$ $\mathrm{mol} / \mathrm{L}$ ); $\mathrm{P}$ e K extraídos pelo $\mathrm{HCl} 0,05 \mathrm{~mol} / \mathrm{L}+\mathrm{H}_{2} \mathrm{SO}_{4}$ $0,0125 \mathrm{~mol} / \mathrm{L}$; e $\mathrm{H}+\mathrm{Al}$ determinados com acetato de cálcio $0,5 \mathrm{~mol} / \mathrm{L}(\mathrm{pH} 7,0)$. O S-SO ${ }_{4}{ }^{2-}$ foi extraído com $\mathrm{Ca}\left(\mathrm{H}_{2} \mathrm{PO}_{4}\right)_{2}, 500 \mathrm{mg} / \mathrm{L}$ de $\mathrm{P}$ em ácido acético $2 \mathrm{~mol} / \mathrm{L}$.

As características químicas do solo foram submetidas à análise de variância. Os efeitos de doses de calcário, gesso e $\mathrm{P}$ foram descritos por meio de equações de regressão. A escolha do modelo foi feita, primeiramente, considerando-se a significância dos coeficientes da regressão e, posteriormente, os coeficientes de determinação, testados pelo teste "t", a 1, 5 e $10 \%$ de probabilidade.

\section{RESULTADOS E DISCUSSÃO}

Houve ajuste de equações de regressão para os efeitos de doses de calcário sobre os teores de $\mathrm{Ca}^{2+}, \mathrm{Al}^{3+}$ e a capacidade de troca de cátions a $\mathrm{pH} 7,0(\mathrm{~T})$ na camada de $0-15 \mathrm{~cm}$ (Tabela 2). $\mathrm{O} \mathrm{pH}$ do solo, na camada de $0-15 \mathrm{~cm}$, aumentou de 5,0 $(1.175 \mathrm{~kg} / \mathrm{ha}$ de calcário ou $25 \%$ da QC) para $5,5(3.525 \mathrm{~kg} / \mathrm{ha}$ de calcário ou $75 \%$ da QC) e, na camada de $15-30 \mathrm{~cm}$, de 4,6 (25\% da QC) para 4,8 (75\% da QC). Nessa camada, não houve

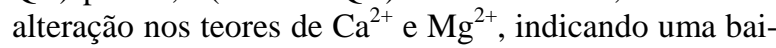

xa movimentação do calcário. Carvalho et al. (1992) afirmam que a movimentação de $\mathrm{Ca}^{2+}$ e $\mathrm{Mg}^{2+}$, adicionados por meio do calcário, no perfil do solo, é um fenômeno conhecido, mas sua intensidade é de difícil previsão, pois, segundo a literatura, é controlada por vários fatores, entre eles a quantidade de água que se move através do perfil, textura do solo, dose e granulometria do calcário, os teores iniciais de $\mathrm{Ca}$ e $\mathrm{Mg}$ no solo, as quantidades e as fontes dos fertilizantes.

Houve efeito significativo da aplicação de gesso sobre os teores de $\mathrm{Ca}^{2+}, \mathrm{Mg}^{2+}$ e $\mathrm{Al}^{3+}, \mathrm{S}_{-} \mathrm{SO}_{4}{ }^{2-}, \mathrm{H}+\mathrm{Al}$, CTC efetiva ( $\mathrm{t}$ ), soma de bases (SB) e porcentagem de saturação por bases (V) e por alumínio (m) no solo, na camada de 0-15 cm. Na camada de $15-30 \mathrm{~cm}$, o efeito foi significativo apenas para os teores de $\mathrm{Al}^{3+}$ e $\mathrm{S}_{-} \mathrm{SO}_{4}{ }^{2-}$ (Tabela 3).

A redução nos teores de $\mathrm{Ca}^{2+}$ na camada de 0-15 cm (Tabela 3) e a tendência de aumento na camada de $15-30 \mathrm{~cm}\left(0,8 ; 0,7 ; 0,7 ; 0,9\right.$ e $\left.1,3 \mathrm{cmol}_{\mathrm{c}} / \mathrm{dm}^{3}\right)$ indicam movimentação de cálcio no perfil do solo com o aumento das doses de gesso.

Na camada de $15-30 \mathrm{~cm}$, o teor médio de $\mathrm{Ca}^{2+}$, $0,9 \mathrm{cmol}_{\mathrm{c}} / \mathrm{dm}^{3}$, é maior que o teor inicial no solo, de 0,3 $\mathrm{cmol}_{\mathrm{c}} / \mathrm{dm}^{3}$, o que representa aumento de $0,6 \mathrm{cmol}_{\mathrm{c}} / \mathrm{dm}^{3}$ de $\mathrm{Ca}^{2+}$ nessa camada de solo. Ressalta-se que pequenos incrementos nos teores de $\mathrm{Ca}^{2+}$ são bastante expressivos, em solos com baixa capacidade de troca de cátions (SILVA et al., 1998).

De fato, vários trabalhos têm demonstrado elevação nos teores de $\mathrm{Ca}^{2+}$ nas camadas superficiais (RECHCIGL e MISLEVY, 1997) e subsuperficiais (DIAS et al., 1994; SMITH et al., 1994; SILVA et al., 1998; WANG et al., 1999) do solo, em função da aplicação de gesso. Silva et al. (1998) verificaram que a substituição de $75 \%$ do $\mathrm{CaCO}_{3}$ pelo $\mathrm{CaSO}_{4}$ aumentou o $\mathrm{Ca}^{2+}$ em 0,30 e $0,68 \mathrm{cmol}_{\mathrm{c}} / \mathrm{dm}^{3}$ nas respectivas camadas de 20-30 e 30-40 cm, em conseqüência da redução dos teores na camada de $0-20 \mathrm{~cm}$ de profundidade.

A redução no teor de $\mathrm{Mg}^{2+}$ na camada de $0-15 \mathrm{~cm}$ (Tabela 3) evidencia a movimentação desse elemento. Segundo Quaggio et al. (1993), as perdas de $\mathrm{Mg}^{2+}$ para as camadas mais profundas do perfil são inversamente proporcionais às doses de calcário aplicadas com o gesso.

As doses crescentes de gesso incrementaram os teores de $\mathrm{S}_{-} \mathrm{SO}_{4}{ }^{2-}$ (Tabela 3), indicando que o gesso é uma excelente fonte de S (FAQUIN et al., 1997). Incrementos maiores foram observados na camada inferior, em decorrência da movimentação de $\mathrm{S}$ na forma de ânion acompanhante. 
TABELA 2 - Equações de regressão das características químicas do solo, nas camadas de 0-15 e 15-30 cm de profundidade em função de doses de calcário (X, kg/ha), após 27 meses das aplicações.

\begin{tabular}{ccc}
\hline Características & \multicolumn{1}{c}{ Equações } \\
\hline $\mathrm{pH}$ & $0-15 \mathrm{~cm}$ & $15-30 \mathrm{~cm}$ \\
$\mathrm{P}\left(\mathrm{mg} / \mathrm{dm}^{3}\right)$ & $\hat{Y}=5,1$ & $\hat{Y}=4,6$ \\
$\mathrm{~S}\left(\mathrm{mg} / \mathrm{dm}^{3}\right)$ & $\hat{Y}=17,4$ & $\hat{Y}=9,2$ \\
$\mathrm{~K}\left(\mathrm{mg} / \mathrm{dm}^{3}\right)$ & $\hat{Y}=16,8$ & $\hat{Y}=21,1$ \\
$\mathrm{Ca}^{2+}\left(\mathrm{cmol}_{\mathrm{c}} / \mathrm{dm}^{3}\right)$ & $\hat{Y}=70,0$ & $\hat{Y}=38,0$ \\
$\mathrm{Mg}^{2+}\left(\mathrm{cmol}_{\mathrm{c}} / \mathrm{dm}^{3}\right)$ & $\hat{Y}=0,668+0,00042^{*} \mathrm{X} ; \mathrm{R}^{2}=0,82$ & $\hat{Y}=0,65$ \\
$\mathrm{Al} 1^{3+}\left(\mathrm{cmol}_{\mathrm{c}} / \mathrm{dm}^{3}\right)$ & $\hat{Y}=1,363-0,00059^{*} \mathrm{X}+0,000000076^{*} \mathrm{X}^{2} ; \mathrm{R}^{2}=0,99$ & $\hat{Y}=0,22$ \\
$\mathrm{H}+\mathrm{Al}\left(\mathrm{cmol}_{\mathrm{c}} / \mathrm{dm}^{3}\right)$ & $\hat{Y}=6,9$ & $\hat{Y}=0,80$ \\
$\mathrm{t}\left(\mathrm{cmol}_{\mathrm{c}} / \mathrm{dm}^{3}\right)$ & $\hat{Y}=2,8$ & $\hat{Y}=6,80$ \\
$\mathrm{~T}\left(\mathrm{cmol}_{\mathrm{c}} / \mathrm{dm}^{3}\right)$ & $\hat{Y}=5,569+0,0026 * \mathrm{X}-0,000000349 * \mathrm{X}^{2} ; \mathrm{R}^{2}=0,99$ & $\hat{Y}=1,70$ \\
$\mathrm{SB}\left(\mathrm{cmol}_{\mathrm{c}} / \mathrm{dm}^{3}\right)$ & $\hat{Y}=2,2$ & $\hat{Y}=7,80$ \\
$\mathrm{~V}(\%)$ & $\hat{Y}=26,0$ & $\hat{Y}=1,03$ \\
$\mathrm{~m}(\%)$ & $\hat{Y}=17,5$ & $\hat{Y}=12,9$ \\
\hline
\end{tabular}

*Significativo a $5 \%$ de probabilidade, pelo teste $t$.

A aplicação de doses crescentes de gesso e decrescentes de calcário foi menos eficiente na redução dos teores de $\mathrm{Al}^{3+}, \mathrm{H}+\mathrm{Al}$ e os valores de m (Tabela 3 ), demonstrando que o $\mathrm{CaCO}_{3}$ é mais eficiente que o $\mathrm{CaSO}_{4}$ na neutralização do $\mathrm{Al}^{3+}$ (WANG et al., 1999). Os valores de $\mathrm{pH}$, na camada de $0-15 \mathrm{~cm}$ do solo, reduziram-se de 5,3 para 5,0 e, na camada de $15-30 \mathrm{~cm}$ do solo, de 5,1 para 4,6, quando comparados nas doses de 0 e $2.820 \mathrm{~kg} / \mathrm{ha}$ de gesso, respectivamente. Por outro lado, reduções no teor de $\mathrm{Al}^{3+}$ e elevações no $\mathrm{pH}$ do solo com o uso do gesso têm sido constatadas, as quais se justificam pelo deslocamento de $\mathrm{OH}^{-}$por $\mathrm{SO}_{4}{ }^{2-}$ (SILVA et al., 1998; WANG et al., 1999). Em solos com predominância de $\mathrm{Fe}$ e Al, Wang et al. (1999) observaram reduções nos teores de $\mathrm{Al}^{3+}$; porém, em solos com t mais elevada e com predominância de cargas permanentes, o $\mathrm{Al}^{3+}$ elevou-se em resposta à aplicação de gesso. Segundo esses autores, em condições de campo, a resposta às aplicações de gesso no solo ainda não está bem elucidada, necessitando, portanto, de definir proporções adequadas de calcário/gesso para cada tipo de solo.

A CTC efetiva e a porcentagem de saturação por bases do solo reduziram-se (Tabela 3 ) certamente em razão da aplicação de doses crescentes de sulfato e decrescentes de carbonato, evidenciando a melhor eficiência do calcário na redução da acidez e no aumento de t. Smith et al. (1994), em solo sob pastagem nativa, observaram que o aumento do $\mathrm{pH}$ do solo, proporcionado pela aplicação de calcário mais gesso, elevou a t do solo, limitando a lixiviação de $\mathrm{Ca}^{2+}$. 
TABELA 3 - Equações de regressão das diferentes características do solo, nas camadas de 0-15 e 15-30 cm de profundidade, em função das doses de gesso (X, kg/ha), após 27 meses das aplicações.

\begin{tabular}{|c|c|c|}
\hline \multirow{2}{*}{$\begin{array}{l}\text { 1/Variáveis } \\
\text { dependentes }\end{array}$} & \multicolumn{2}{|c|}{ Camadas de solo (cm) } \\
\hline & $0-15$ & $15-30$ \\
\hline $\mathrm{pH}$ & $\hat{Y}=5,1$ & $\hat{Y}=4,7$ \\
\hline $\mathrm{P}$ & $\hat{Y}=18,5$ & $\hat{Y}=9,6$ \\
\hline S & $\hat{Y}=13,46+0,439 * \mathrm{X}^{0,5}-0,0057 * * * \mathrm{X} ; \mathrm{R}^{2}=0,98$ & $\hat{Y}=15,90+0,495 * \mathrm{X}^{0,5}-0,0071 * * * \mathrm{X} ; \mathrm{R}^{2}=0,94$ \\
\hline K & $\hat{Y}=60,8$ & $\hat{Y}=37,4$ \\
\hline $\mathrm{Ca}^{2+}$ & $\hat{Y}=3,53-0,0913 * \mathrm{X}^{0,5}+0,0011 * \mathrm{X} ; \mathrm{R}^{2}=0,99$ & $\hat{Y}=0,9$ \\
\hline $\mathrm{Mg}^{2+}$ & $\hat{Y}=0,85-0,00017 * \mathrm{X} ; \mathrm{R}^{2}=0,75$ & $\hat{Y}=0,6$ \\
\hline $\mathrm{Al}^{3+}$ & $\hat{Y}=0,16+0,00014 * \mathrm{X} ; \mathrm{R}^{2}=0,94$ & $\hat{Y}=0,38+0,017 * * * \mathrm{X}^{0,5}-0,0002 * * * \mathrm{X} ; \mathrm{R}^{2}=0,97$ \\
\hline $\mathrm{H}+\mathrm{Al}$ & $\hat{Y}=5,96+0,00067 * \mathrm{X} ; \mathrm{R}^{2}=0,72$ & $\hat{Y}=6,9$ \\
\hline $\mathrm{t}$ & $\hat{Y}=4,78-0,1049 * * * \mathrm{X}^{0,5}+0,0013^{\mathrm{NS}} \mathrm{X} ; \mathrm{R}^{2}=0,98$ & $\hat{Y}=2,0$ \\
\hline $\mathrm{T}$ & $\hat{Y}=9,6$ & $\hat{Y}=8,2$ \\
\hline SB & $\hat{Y}=4,67-0,1122 * * * \mathrm{X}^{0,5}+0,0013^{\mathrm{NS}} \mathrm{X} ; \mathrm{R}^{2}=0,98$ & $\hat{Y}=1,5$ \\
\hline $\mathrm{V}$ & $\hat{Y}=45,89-0,9593 * * * \mathrm{X}^{0,5}+0,0103^{\mathrm{NS}} \mathrm{X} ; \mathrm{R}^{2}=0,96$ & $\hat{Y}=16,6$ \\
\hline $\mathrm{m}$ & $\hat{Y}=6,15+0,00524 * \mathrm{X} ; \mathrm{R}^{2}=0,88$ & $\hat{Y}=33,2$ \\
\hline
\end{tabular}

*,***Significativo a 5 e $10 \%$ de probabilidade, pelo teste $t$; NS não-significativo

${ }^{1 /} \mathrm{P}, \mathrm{S}, \mathrm{K} \mathrm{em} \mathrm{mg/dm}{ }^{3} ; \mathrm{Ca}^{2+}, \mathrm{Mg}^{2+} ; \mathrm{Al}^{3+} ; \mathrm{H}+\mathrm{Al}, \mathrm{t} \mathrm{e} \mathrm{T} \mathrm{em} \mathrm{cmol}_{\mathrm{c}} / \mathrm{dm}^{3} ; \mathrm{V} \mathrm{e} \mathrm{m} \mathrm{em} \mathrm{\% .}$

No primeiro ano de avaliação, as aplicações de gesso e de calcário não tiveram efeito significativo $(\mathrm{P}>0,05)$ na densidade de perfilhos e altura de plantas. No segundo ano, embora tenham proporcionado aumento na densidade de perfilhos e na altura de plantas do capimbraquiária, aumentaram a participação da leguminosa no consórcio (Tabela 4). Aumentos nos teores de cálcio $\left(\hat{Y}=1,087+0,00010^{* *} \mathrm{G} ; \mathrm{R}^{2}=0,87 ; \hat{Y} \mathrm{em} \mathrm{dag} / \mathrm{kg}\right)$, potássio $\left(\hat{Y}=0,712+0,00011 * * \mathrm{G} ; \mathrm{R}^{2}=0,82 ; \hat{Y} \mathrm{em}\right.$ dag/kg) e enxofre $\left(\hat{Y}=0,061+0,000023 * * \mathrm{G} ; \mathrm{R}^{2}=0,89\right.$; $\hat{Y}$ em dag/kg) na matéria seca do estilosantes demonstram melhoria no seu estado nutricional, o que pode ter contribuído para aumentar a porcentagem da leguminosa na pastagem. Delistoianov et al. (1992) constataram que a aplicação de $45 \mathrm{~kg} / \mathrm{ha}$ de $\mathrm{S}$, sob a forma de gesso, elevou os teores de $\mathrm{S}$ em leguminosas e, segundo esses autores, o $\mathrm{S}$ aumenta a persistência da leguminosa na pastagem, pois estimula a nodulação e a fixação de $\mathrm{N}_{2}$.

As doses de $\mathrm{P}_{2} \mathrm{O}_{5}$ elevaram os teores de $\mathrm{P}$ no solo, na camada de $0-15 \mathrm{~cm}(\hat{Y}=-3,156+0,2452 * * \mathrm{X}$; $\mathrm{R}^{2}=0,87 ; \hat{Y}$ em $\mathrm{mg} / \mathrm{dm}^{3}$ ) e estimaram aumentos de $0,2452 \mathrm{mg} / \mathrm{dm}^{3}$ de $\mathrm{P}$ por $\mathrm{kg} / \mathrm{ha}$ de $\mathrm{P}_{2} \mathrm{O}_{5}$ aplicado. Assim, para recomendação de $100 \mathrm{~kg} / \mathrm{ha}$ de $\mathrm{P}_{2} \mathrm{O}_{5}$ (CFSEMG, 1999), estima-se teor de $\mathrm{P}$ disponível nesse solo da ordem de $24,0 \mathrm{mg} / \mathrm{dm}^{3}$, embora, sem ajuste de regressão, os teores de $\mathrm{Ca}^{2+}(2,0 ; 1,1 ; 1,9 ; 2,7$ e 3,1 $\left.\mathrm{cmol}_{\mathrm{c}} / \mathrm{dm}^{3}\right)$ e a soma de bases $(2,9 ; 3,2 ; 3,1 ; 3,7$ e 4,3 $\mathrm{cmol}_{\mathrm{c}} / \mathrm{dm}^{3}$ ) na camada de $0-15 \mathrm{~cm}$ tenderam a aumentar e os teores de $\mathrm{Al}^{3+}$ tenderam a reduzir $(0,2 ; 0,3 ; 0,2 ; 0,1$ e $0,1 \mathrm{cmol}_{\mathrm{c}} / \mathrm{dm}^{3}$ ), com as doses de $\mathrm{P}$, o que também foi 
verificado por Mears et al. (1993) e Leite et al. (1996). Provavelmente, esses acréscimos ocorreram em razão da presença de Ca na composição química do superfosfato triplo.

Houve redução na porcentagem de estilosantes, em decorrência do maior perfilhamento e da maior altura de plantas da gramínea (Tabela 5), reduzindo a competição da leguminosa. Por outro lado, a baixa freqüência de cortes nas forrageiras, três cortes no período de primavera/verão com intervalos de 48 dias, certamente contribuiu para maior competição da gramínea com a leguminosa. Desse modo, a aplicação de doses mais elevadas de $\mathrm{P}$, em sistemas de baixa utilização da forragem ou com intervalos de corte mais amplos, podem aumentar a competição da gramínea e reduzir a porcentagem do estilosantes na pastagem. Sugere-se que a aplicação de doses de P diferenciadas e localizadas para a gramínea e para a leguminosa pode ser uma alternativa para redução da competição da gramínea.

TABELA 4 - Equações de regressão para a porcentagem de leguminosa no consórcio, para a densidade de perfilhos (perfilhos $/ \mathrm{m}^{2}$ ), altura de plantas e produção de matéria seca (MS) de capim-braquiária(MS), em função de doses de gesso ( $\mathrm{G}, \mathrm{kg} / \mathrm{ha})$.

\begin{tabular}{ccc}
\hline Variáveis dependentes & Equações & $\mathbf{R}^{\mathbf{2}}$ \\
\hline Leguminosa (\%) & $2^{\text {o Ano }(1998 / 1999)}$ & 0,79 \\
Perfilhos $/ \mathrm{m}^{2}$ & $\hat{Y}=13,42+0,0024443^{*} \mathrm{G}$ & 0,80 \\
Altura (cm) & $\hat{Y}=514,0+0,0148833^{* *} \mathrm{G}$ & 0,86 \\
MS (kg/ha) & $\hat{Y}=83,92+0,0052492^{* * \mathrm{G}}$ & 0,72 \\
\hline
\end{tabular}

\section{*,** Significativo a 5 e $1 \%$ de probabilidade, pelo teste $t$.}

TABELA 5 - Equações de regressão para a porcentagem de leguminosa no consórcio, para a densidade de perfilhos (perfilhos $/ \mathrm{m}^{2}$ ), altura de plantas e produção de matéria seca (MS) de capim-braquiária, em função de doses de $\mathrm{P}_{2} \mathrm{O}_{5}$ (P, kg/ha).

\begin{tabular}{ccc}
\hline Variáveis dependentes & Equações & $\mathbf{R}^{2}$ \\
\hline Leguminosa (\%) & $1^{\mathrm{o}}$ Ano $(1997 / 1998)$ & 0,80 \\
Perfilhos/m ${ }^{2}$ & $\hat{Y}=21,11-0,0816485^{* * \mathrm{P}}$ & 0,95 \\
Altura (cm) & $\hat{Y}=303,49+1,26309 * * \mathrm{P}$ & 0,97 \\
MS (kg/ha) & $\hat{Y}=68,19+0,0970909 * * \mathrm{P}$ & 0,82 \\
\hline Leguminosa $(\%)$ & $\hat{Y}=352,1+10,4473 * * \mathrm{P}$ & 0,92 \\
Perfilhos/m ${ }^{2}$ & $2^{\circ}$ Ano $(1998 / 1999)$ & 0,95 \\
Altura (cm) & $\hat{Y}=29,34-0,0967167 * * \mathrm{P}$ & 0,96 \\
MS (kg/ha) & $\hat{Y}=446,81+0,588889 * * \mathrm{P}$ & - \\
\hline
\end{tabular}

\footnotetext{
** Significativo a $1 \%$ de probabilidade, pelo teste $\mathrm{t}$.
} 


\section{CONCLUSÕES}

a) A substituição do calcário pelo gesso melhorou o ambiente radicular, translocando $\mathrm{Ca}^{2+}, \mathrm{Mg}^{2+}$ e S$\mathrm{SO}_{4}{ }^{2-}$ para a camada de $15-30 \mathrm{~cm}$ de profundidade.

b) A substituição de $37,5 \%$ do $\mathrm{CaO}$ do calcário pelo $\mathrm{CaO}$ do gesso propiciou a maior porcentagem de estilosantes na pastagem.

c) A aplicação de $\mathrm{P}$ aumentou o teor de $\mathrm{P}$ disponível no solo na camada de $0-15 \mathrm{~cm}$ e favoreceu o crescimento da gramínea.

\section{RERERÊNCIAS BIBLIOGRÁFICAS}

ANDRADE, R. P.; KARIA, C. T. Uso de Stylosanthes em pastagens no Brasil. In: SIMPÓSIO DE FORRAGICULTURA E PASTAGENS, 1., 2000, Lavras. Anais... Lavras: DZO/UFLA, 2000. p. 273-309.

CARVALHO, M. M.; MARTINS, C. E.; SIQUEIRA, C.; SARAIVA, O. F. Crescimento de uma espécie de braquiária, na presença da calagem em cobertura e de doses de nitrogênio. Revista Brasileira de Ciência do Solo, Viçosa, v. 16, p. 69-74, 1992.

COMISSÃO DE FERTILIDADE DO SOLO DO ESTADO DE MINAS GERAIS. Recomendações para o uso de corretivos e fertilizantes em Minas Gerais: 5ª Aproximação. Viçosa: UFV, 1999. 359 p.

DELISTOIANOVM J.; MATTOS, H. B.; MONTEIRO, F. A. Aplicação de fontes de fósforo e gesso em uma pastagem consorciada estabelecida em um Latossolo Vermelho-Amarelo. Boletim da Indústria Animal, v. 49, n. 2, p. 83-90, 1992.

DIAS, L. E.; ALVAREZ, V. H.; COSTA, L. M.; NOVAIS, R. F. Distribuição de algumas formas de enxofre em colunas de solos tratados com diferentes doses de fósforo e gesso. Revista Brasileira de Ciência do Solo, Viçosa, v. 18, p. 373-380, 1994.

DIOGO, J. M. S.; NASCIMENTO JÚNIOR, D.; SANCHES, L. J. T. Composição botânica da dieta selecionada por novilhos em pastagem natural de Viçosa MG. Revista Brasileira de Zootecnia, Viçosa, v. 24, n. 6, p. 884-895, nov./dez. 1995.

EMPRESA BRASILEIRA DE PESQUISA AGROPECUÁRIA. Recomendações para estabelecimento e utilização do Stylosanthes guianensis cv. Mineirão. Planaltina, 1993. 6 p. (EMBRAPA-CPAC, Comunicado Técnico, 67).
FAQUIN, V.; PASSOS, R. R.; VILLA, M. R.; CURI, N.; EVANGELISTA, A. R. Absorção e acumulação de nutrientes por gramíneas forrageiras sob influência de fontes de fósforo e correção do solo. Revista Brasileira de Zootecnia, Viçosa, v. 26, n. 2, p. 219-226, mar./abr. 1997.

GONÇALVES, C. A.; CAMARÃO, A. P.; SIMÃO NETO, M.; DUTRA, S. Consorciação de gramíneas e leguminosas forrageiras com e sem fertilização fosfatada no nordeste paraense, Pará. In: REUNIÃO DA SOCIEDADE BRASILEIRA DE ZOOTECNIA, 34., 1997, Juiz de Fora. Anais... Juiz de Fora: SBZ, 1997. p. 42-44.

LEITE, V. B. O.; MONTEIRO, F. A.; WERNER, J. C. Influência da calagem e da fertilização com fósforo e molibdênio sobre o cultivo da associação green-panic $\mathrm{x}$ galáxia em solo de cerrado. Boletim da Indústria Animal, Nova Odessa, v. 53, p. 71-82, 1996.

MEARS, P. T.; HAVILAH, E. J.; PRICE, G. H.; DARNELL, R. E.; McLENNAN, D. J.; WILSON, C. Superphosphate maintains soil fertility and beef production on grazed white clover pastures in the subtropics. 1: residual soil phosphorus, sulfur, and nitrogen, and pasture growth responses. Australian Journal of Experimental Agriculture, Melbourne, v. 33, n. 4, p. 425-434, 1993.

MICHALK, D. L.; NAN-PING, F.; CHIN-MING, Z. Improvement of dry tropical rangelands on Hainan Island, China. 4. Effect of seedbed on pasture establishment. Journal of Range Management, [S.1.], v. 51, n. 1, p. 280-288, Jan. 1988.

PACHECO, B. M.; NASCIMENTO JÚNIOR, D.; REGAZZI, A. J. Comparação de métodos para estimativas da composição botânica de pastagem. Revista Brasileira de Zootecnia, Viçosa, v. 16, n. 4, p. 337351, jul./ago. 1987.

QUAGGIO, J. A.; RAIJ, B. van; GALLO, P. B.; MASCARENHAS, A. A. Respostas da soja à aplicação de calcário e gesso e lixiviação de íons no perfil do solo. Revista Brasileira de Zootecnia, Viçosa, v. 28, n. 3, p. 375-383, mar. 1993.

RECHCIGL, J. E.; MISLEVY, P. Stargrass response to lime and phosphogypsum. Journal of Production Agricultural, [S.1.], v. 10, n. 1, p. 101-105, Jan. 1997. 
SANTOS, M. V. F.; NASCIMENTO JÚNIOR, D.; PEREIRA, J. C.; REGAZZI, A. J.; SILVA, A. G.; DIOGO, J. M. S. Composição florística, densidade e altura de uma pastagem natural sob pastejo. Revista Brasileira de Zootecnia, Viçosa, v. 27, n. 6, p. 1082-1091, nov./dez. 1998.

SILVA, A. A.; VALE, F. R.; FERNANDES, L. A.; FURTINI NETO, A. E.; MUNIZ, J. A. Efeitos de relações $\mathrm{CaSO}_{4} / \mathrm{CaCO}_{3}$ na mobilidade de nutrientes no solo e no crescimento do algodoeiro. Revista Brasileira de Ciência do Solo, Campinas, v. 22, p. 451-457, 1998.

SMITH, C. J.; PEOPLES, M. B.; KEERTHYSINGHE, G.; JAMES, T. R.; GARDEN, D. L.; TUOMI, S. S. Effect of surface applications of lime, gypsum and phosphogypsum on the alleviating of surface and subsurface acidity in a soil under pasture. Australian Journal of Soil Research, Melbourne, v. 32, p. 9951008, 1994.

WANG, H. L.; HEDLEY, M. J.; BOLAN, N. S.; HORNE, D. J. The influence of surface incorporated lime and gypsiferous by-products on surface and subsurface soil acidity. 1. Soil solution chemistry. Australian Journal of Soil Research, Melbourne, v. 37, p. 165-180, 1999.

ZIMMER, A. H.; MACHADO, M. C. M.; BARCELOS, A. O.; KICHEL, A. N. Estabelecimento e recuperação de pastagens de Brachiaria. In: SIMPÓSIO SOBRE MANEJO DA PASTAGEM, 11., 1994, Piracicaba. Anais... Piracicaba: FEALQ/ESALQ, 1994. p. 153-208. 